\title{
La atención prenatal en la ciudad fronteriza de Tijuana, México
}

\author{
M. Ramírez-Zetina, ${ }^{1}$ V. Richardson, ${ }^{2}$ H. Ávila, ${ }^{3}$ V. E. Caraveo, ${ }^{3}$ \\ R. E. Salomón, ${ }^{4}$ M. Bacardíi y A. Jiménez-Cruz ${ }^{4}$
}

RESUMEN El presente estudio se diseñó con el fin de conocer las condiciones en las que se realiza el control prenatal en la ciudad fronteriza de Tijuana, Baja California, México y de estudiar las posibles asociaciones entre ese tipo de atención y el resultado neonatal en términos de peso al nacer, estado de salud del neonato y prematuridad. Se seleccionaron siete hospitales que atienden a personas de diferentes estratos socioeconómicos y entre diciembre de 1993 y marzo de 1994 se entrevistó en ellos a 279 mujeres que cursaban las primeras 24 a 48 horas del puerperio. En la entrevista se recogieron datos sobre nivel socioeconómico; conceptos, actitudes y prácticas de las madres respecto de la salud obstétrica; percepción materna de acceso a los servicios de atención prenatal; calidad de las consultas de control prenatal (evaluada según la realización de pruebas de sangre y orina y la medición de la tensión arterial y del peso); y antecedentes ginecobstétricos y de salud de la madre. Se elaboró una base de datos con el paquete estadístico SPSS y se exploraron posibles asociaciones entre la atención prenatal como variable independiente y las diferentes variables dependientes mediante tablas de contingencia y la prueba de Fisher de dos colas.

No se encontraron neonatos prematuros, enfermos o con un peso al nacer $\leq 2500 \mathrm{~g}$. Por este último motivo se optó por dicotomizar la variable correspondiente al peso al nacer en $\leq 3000 \mathrm{~g}$ $y>3000 \mathrm{~g}$. Se observó una asociación significativa $(\mathrm{P}<0,00038)$ entre la ausencia de control prenatal y un menor peso al nacer. La ausencia de control prenatal a su vez se asoció con bajos ingresos familiares; dependencia económica materna; convivencia de la madre en unión libre; poca comunicación con la pareja; falta de seguro médico; presencia de un embarazo no deseado; y haber acudido al Hospital General para la atención del parto. De la muestra total $(\mathrm{n}=15)$, $5,4 \%$ no recibió atención prenatal. Ninguna de las mujeres que no recibieron atención declaró haber enfrentado dificultades que le impidieran el acceso, pero de las 15 solamente 7 contestaron la pregunta. Se encontró que la atención prenatal era de buena calidad en $68 \%$ de los casos $(\mathrm{n}=190)$ y que tanto el número de las consultas como el momento en que se llevaron a cabo fueron adecuados en $50 \%$ de los casos $(\mathrm{n}=142)$. El haber trabajado durante el embarazo o en algún momento previo al embarazo tuvo valor predictivo positivo en cuanto a la utilización de los servicios de atención prenatal. Hacen falta estudios adicionales para evaluar la posible relación entre la violencia doméstica y la ausencia de cuidados prenatales o la demora en buscarlos.

1 Instituto Mexicano del Seguro Social, Tijuana, Baja California, México. Dirección postal: Calle 5 ta. Esq. con Avenida " $F$ ", Zona Centro, Tijuana, Baja California, México. C.P. 22,000.

2 Hospital del Instituto de Seguridad y Servicios Sociales de los Trabajadores del Gobierno y Municipios del Estado de Baja California (ISSSTECALI), Tijuana, Baja California, México.

3 Instituto Nacional de Perinatología, Dirección de Investigación en Salud Pública, México, D.F.

4 Universidad Autónoma de Baja California, Facultad de Medicina, Investigación y Docencia, Tijuana, Baja California, México.
La salud maternoinfantil continúa siendo uno de los grandes retos en el campo de la salud pública. En los proyectos que forman parte de la Revisión de la Atención Primaria de la Salud (RAPS) realizados en el contexto de la región fronteriza entre México y los Estados Unidos a principios de la década 1990-2000, la atención mater- noinfantil fue identificada como una de las áreas de mayor interés para la investigación colectiva binacional, junto con la salud del adolescente y la salud ambiental y ocupacional (1). Aun cuando no se ha logrado llegar a un consenso sobre el número ideal o mínimo de consultas prenatales $(2,3)$, se reconoce que tanto la frecuencia 
como la calidad de la atención prenatal afectan a la supervivencia infantil.

Tijuana, Baja California, México, es una ciudad fronteriza con un millón de habitantes y casi medio millón de población flotante (4). Colinda hacia el norte con California, el estado más rico de los Estados Unidos, y representa para los latinoamericanos una vía de paso hacia el vecino país. Como en la mayoría de los países en desarrollo, la mortalidad perinatal en Tijuana es alta y en 1997 la tasa de mortalidad perinatal registrada fue de 21,1 defunciones por 1000 nacidos vivos. Según los resultados de uno de los escasos estudios sobre la mortalidad infantil en Tijuana, más de la mitad $(61,12 \%)$ de las muertes infantiles en esta ciudad ocurren durante el período perinatal I (entre la semana 28 de la gestación y el día 7 después del nacimiento) (5). A pesar de haberse detectado una correlación entre la atención prenatal y el resultado del embarazo (6-8), aún hay poco conocimiento sobre los factores que contribuyen a la utilización de los servicios de atención prenatal y sus características en Tijuana.

Estudios norteamericanos realizados en la población hispana que reside cerca de la frontera con México coinciden en señalar que en esa zona las mujeres que han nacido fuera de los Estados Unidos y particularmente las que tienen poco tiempo de haber inmigrado o las que hablan español exclusivamente, suelen tener una baja cobertura de atención prenatal por motivos culturales, económicos y educacionales y por influencia de factores propios del sistema de salud. Entre estos últimos figuran los largos tiempos de espera y la pobre relación entre médico y paciente (9-11).

La presente investigación tuvo los siguientes objetivos: 1) examinar la calidad de la atención prenatal en Tijuana; 2) calificar el resultado neonatal en función del peso al nacer, el estado de salud del neonato y la prematuridad y 3) estudiar las posibles asociaciones entre la calidad de la atención prenatal, el resultado neonatal y los siguientes factores: a) ingreso semanal en el hogar de la madre; b) tipo de institución hospitalaria donde se atendió el parto (de la seguridad social, pública o privada); c) conceptos, actitudes y prácticas de las madres en relación con la salud obstétrica y d) acceso a los servicios de salud.

\section{MATERIALES Y MÉTODOS}

Se seleccionaron siete hospitales de la ciudad de Tijuana que atienden a personas de diferentes estratos sociales. El proceso de muestreo comprendió dos etapas. En la primera se efectuó un muestreo de conveniencia por conglomerados para seleccionar siete hospitales: el Instituto Mexicano del Seguro Social (IMSS) y el Instituto de Seguridad y Servicios Sociales de los Trabajadores del Gobierno y Municipios del Estado de Baja California (ISSSTECALI); el Hospital General, que es público, y cuatro hospitales privados. Para este muestreo se tuvo en cuenta la disponibilidad de sujetos durante el período de estudio. La segunda etapa consistió en un muestreo por cuotas, eligiéndose en cada institución sujetos de estudio en proporción con el número de mujeres cuyos partos fueron atendidos en cada conglomerado bajo observación. Durante el período comprendido entre diciembre de 1993 y marzo de 1994, se entrevistó a 279 mujeres que cursaban las primeras 24 a 48 horas del puerperio. El instrumento de medición, que contenía 106 variables, se validó mediante una prueba de campo. De los expedientes clínicos hospitalarios se obtuvieron datos sobre el neonato y la progresión del parto.

Los ingresos familiares semanales se clasificaron en cinco categorías - 0 a 200; 201 a 500; 501 a $1000 ; 1001$ a 1500 y más de 1500 nuevos pesos mexicanos- y su análisis se basó en los datos obtenidos en la entrevista. El resultado neonatal se valoró sobre la base de lo registrado en el expediente clínico intrahospitalario, de acuerdo con los siguientes rubros y sus correspondientes parámetros: bajo peso al nacer $(<2500$ g); prematuridad ( $<$ de 37 semanas de gestación) y estado de salud del neonato. El estado de salud o enfermedad del neonato se evaluó según la ausencia o presencia, respectivamente, de los siguientes trastornos durante el parto: 1) sufrimiento fetal agudo (taquicardia o bradicardia fetal), presencia de meconio grueso y Apgar menor de 6 al minuto, a los 5 minutos del nacimiento, o ambos y b) trastorno neonatal inmediato en forma de dificultad respiratoria, infección, malformación congénita y encefalopatía hipóxica isquémica.

Para calificar la calidad de la atención prenatal se construyó un índice basado en cuatro criterios: medición de la tensión arterial; medición del peso corporal; toma de muestra de orina y toma de muestra de sangre. Durante la entrevista se investigó la frecuencia con que se realizaron los dos primeros procedimientos en las consultas prenatales, asignándose dos puntos si se habían realizado en todas las consultas, 1 punto si se habían realizado en algunas de las consultas y cero puntos si nunca se habían realizado. En el caso de las muestras de sangre y orina, se preguntó si se habían obtenido en cualquier momento de la atención médica prenatal, asignándose 2 puntos si la respuesta era afirmativa y cero si era negativa. Se consideró "buena" la atención de una paciente con un total de 7-8 puntos; "regular" la de una paciente con un total de 4-6 puntos, y "deficiente" la de una paciente con un total de 0-3 puntos.

Se investigó la presencia de una correlación entre el momento de la primera consulta y el número total de consultas prenatales, aplicando una adaptación de los criterios utilizados por el "Comité de Estudio para la Prevención del Bajo Peso al Nacer" del Instituto de Medicina de Estados Uni$\operatorname{dos}(6)$.

Se elaboró una base de datos con el programa estadístico SPSS y se analizaron posibles asociaciones entre la variable dependiente (el resultado neonatal) y las variables independientes: calidad de la atención prenatal; ingreso semanal en el hogar de la madre; tipo de institución hospitalaria donde se atendió el parto; conceptos, actitudes y prácticas maternas en relación con la salud obstétrica, y acceso a los servicios de salud, según lo referido por la madre. Todas estas variables fueron exploradas mediante preguntas 
específicas en la entrevista. Las dificultades para tener acceso a la atención prenatal fueron dicotomizadas en presentes y ausentes. El análisis se realizó mediante la construcción de tablas de contingencia y la prueba de hipótesis de Fisher de dos colas.

\section{RESULTADOS}

De las mujeres en las muestras de los conglomerados, 59\% $(n=165)$ fueron atendidas en las instituciones de la seguridad social, $22 \%(n=61)$ en el Hospital General y 19\% $(n=53)$ en los hospitales privados. No se encontraron neonatos prematuros, enfermos o con un peso al nacer $<2500 \mathrm{~g}$, en vista de lo cual se optó por dicotomizar el peso al nacer en peso $\leq 3000 \mathrm{~g} \mathrm{y}>$ 3000 g. El peso promedio fue de 3 267,13 g con una desviación estándar (DE) de 537,59 g. Se observó una asociación estadísticamente significativa entre la ausencia de control prenatal y un peso al nacer $\leq 3000 \mathrm{~g}(P<0,00038)$, hallazgo que llevó al análisis de las otras variables dependientes desde el punto de vista de su posible asociación con la presencia o ausencia de control prenatal.

Del total de 279 mujeres entrevistadas, $15(5,4 \%)$ refirieron no haber recibido atención prenatal. Se encontraron asociaciones estadísticamente significativas entre la ausencia de control prenatal y los siguientes factores: ingreso bajo ${ }^{5}(P<0,007)$, declarado por $19,7 \%$ de las entrevistadas; convivencia en unión libre $(P<0,001)$, y atención del parto en el Hospital General $(P<0,0003)$, que fue recibida por $67 \%$ de las mujeres que no tuvieron control prenatal.

El no haber trabajado durante el embarazo y, más aún, el no haber trabajado nunca también mostraron una asociación significativa con la ausencia de control prenatal $(P<0,03$ y $P<$ $0,016)$. De todas las entrevistadas,

\footnotetext{
5 Menos de 200 nuevos pesos mexicanos semanales, equivalentes a alrededor de US\$ 25 en el momento de la entrevista.
}

$40,1 \%$ indicaron no haber trabajado nunca; $38 \%$ dijeron haber trabajado durante el embarazo y 21,9\% declararon haberlo hecho únicamente antes del embarazo.

La carencia de un seguro médico fue otro factor que se asoció significativamente con la ausencia de control prenatal $(P<0,011)$. A la pregunta sobre la posesión de un seguro médico social o privado, 151 mujeres $(54,1 \%)$ contestaron afirmativamente y $125(44,8 \%)$ negativamente. Tres mujeres no contestaron la pregunta.

Los sentimientos negativos frente a la sospecha de embarazo $(P<0,005)$ y, más aún, los sentimientos negativos al haberse confirmado el embarazo $(P<$ $0,001)$ mostraron una intensa asociación directa con la carencia de atención prenatal. Asimismo, se observó una asociación signficativa entre la ausencia de cuidados prenatales y la falta de interés manifiesto por parte de la pareja en el estado de salud y el embarazo de la mujer $(P<0,001)$. No se encontró asociación significativa entre no vivir con la pareja y la ausencia de control prenatal.

Pese a que 99\% de las mujeres reconocían la importancia de la atención médica durante el embarazo, 5,4\% no la recibieron. Doscientas veintisiete mujeres $(81,4 \%)$ negaron haber enfrentado dificultades o barreras para tener acceso a atención prenatal y 29 $(10,4 \%)$ reconocieron haberlas enfrentado; $23(8,2 \%)$ no contestaron la pregunta. Se observó una discreta asociación $(P<0,04)$ entre la ausencia de dificultades para tener acceso a la atención y un mayor peso al nacer (>3 $000 \mathrm{~g})$, pero curiosamente no se detectó ninguna asociación entre el haber enfrentado o no dificultades y la presencia o ausencia de control prenatal. De hecho, del total de 15 mujeres que no acudieron a control, las siete que contestaron las preguntas sobre el acceso a la atención no refirieron haber enfrentado barreras. Las dificultades mayormente referidas entre las mujeres que recibieron atención se relacionaron con algunos aspectos administrativos de la misma (largos tiempos de espera), seguidos de una falta de apoyo social (para el cuidado de los otros hijos) y, en menor grado, con dificultades económicas.

Haber recibido atención durante el parto en un hospital privado y tener ingresos altos o moderados fueron factores asociados con un mejor peso al nacer, aunque la asociación no fue estadísticamente significativa. Tampoco se observó ninguna asociación significativa entre un menor peso al nacer y la escolaridad u ocupación de la madre o el padre; el haber tenido otros hijos con bajo peso al nacer; la paridad; la edad materna en el momento de la concepción; o el consumo de suplementos vitamínicos. Ninguno de los embarazos observados en esta muestra de mujeres fue de alto riesgo a causa de un trastorno materno subyacente.

Del total de la muestra, 68\% (190 mujeres) recibió una "buena" atención; 16\% (45), una atención "regular" y $12 \%$ (32), una atención "deficiente". Se omitieron del análisis 12 mujeres que no contestaron todas las preguntas sobre los cuatro criterios utilizados para elaborar el índice de calidad y las 15 que no recibieron atención prenatal fueron incluidas en el grupo de atención prenatal "deficiente".

Se encontró que 50\% de las mujeres (142) recibieron una atención adecuada; 25\% (70) recibieron una atención de calidad intermedia, y 14\% (39), recibieron cuidados inadecuados. Veinticuatro casos no recordaban la respuesta a alguna de las dos preguntas y cuatro voluntariamente decidieron no contestar las preguntas. Al no contar con la información, estos casos no se incluyeron en esta sección del análisis. Los 15 casos que no recibieron control prenatal se incluyeron en el grupo de atención inadecuada.

\section{DISCUSIÓN}

Aunque en este estudio no se observó ningún caso de defunción neonatal, bajo peso al nacer ( $<2500 \mathrm{~g})$ o prematuridad, se pudo identificar una asociación significativa entre la ausencia de control prenatal y un menor peso al nacer (<3 $000 \mathrm{~g})$, También se logró definir las características de las mujeres con más alto riesgo de no reci- 
bir atención prenatal: bajos ingresos; dependencia económica; convivencia en unión libre; poca comunicación con la pareja; ausencia de seguro médico; presencia de embarazo no deseado, y parto atendido en el Hospital General.

Estos resultados deben interpretarse cuidadosamente. Primero, aun cuando nuestro modelo reveló asociaciones significativas entre las variables mencionadas y la ausencia de control prenatal, que a su vez mostró una asociación con un peso al nacer menor de $3000 \mathrm{~g}$, esto no demuestra causalidad. Segundo, las variables del modelo se limitaron a las obtenidas mediante la entrevista y a las que se podían encontrar en todos los expedientes clínicos hospitalarios, como el peso al nacer. Mediciones confiables de otras variables que podrían asociarse con el peso del niño, tales como el aumento ponderal durante la gestación, el peso y la talla de la madre antes del embarazo y su estado de salud pregestacional no se habían incluido en todos los expedientes clínicos y por tal motivo no se les tuvo en cuenta. Finalmente, la entrevista fue aplicada en el postparto mediato o tardío, por lo que las respuestas podrían estar sujetas a cierto sesgo de recordación.

Aun a la luz de estas limitaciones, la asociación principal —es decir, entre la ausencia de cuidados prenatales y peso al nacer menor de $3000 \mathrm{~g}$ - fue robusta y coincide con lo notificado en la literatura $(6,8,11)$.

Fueron particularmente interesantes los resultados relacionados con los antecedentes laborales de la mujer. El haber trabajado durante el embarazo o en cualquier momento de la vida previo al embarazo fue factor predictivo positivo de recibir atención. Nuestra revisión bibliográfica no arrojó ningún otro estudio que estableciera una asociación entre el trabajo y la obtención de la atención prenatal, aunque se ha documentado que la mujer que trabaja percibe tener un mayor control sobre su vida (12). Es posible que la expectativa de percibir pago de incapacidad por maternidad lleve a las mujeres trabajadoras que están inscritas en los sistemas de seguridad social a solicitar atención médica. No obstante, las mu- jeres que habían trabajado antes del embarazo se mostraron igualmente propensas a acudir a control prenatal.

Se desconocen los motivos por los cuales ocho de las 15 mujeres que carecieron de cuidados prenatales hayan preferido no contestar las preguntas sobre el acceso a la atención médica. Tampoco se sabe por qué 15 mujeres no acudieron a control prenatal, aun cuando conocían la importancia de hacerlo. Estos puntos deben estudiarse en investigaciones subsecuentes. Según algunos autores, la atención prenatal tardía y la falta de atención podrían deberse a la oposición de la pareja o al temor de que en la exploración médica se descubran huellas de abuso físico en situaciones de violencia doméstica (13). Este aspecto no fue explorado directa o indirectamente en la entrevista, por lo que persiste la interrogante de los motivos que tuvieron estas mujeres para abstenerse de contestar.

A la fecha hay quienes argumentan que la débil asociación entre la atención prenatal y el peso al nacer se atribuye a un control prenatal de mala calidad (14). En el presente estudio más de la mitad de las mujeres recibieron una atención "buena" y la mitad recibió una atención oportuna y un número adecuado de controles médicos prenatales. Quizá ello explique en parte la asociación observada entre el haber recibido atención prenatal y un mejor peso al nacer. Este hallazgo es plausible aun teniendo en cuenta las limitaciones propias de los datos obtenidos por entrevista. La calidad de la atención observada es comparable a la encontrada en otra investigación realizada en México sobre la atención de la mujer embarazada en el contexto de la seguridad social (15).

\section{CONCLUSIONES}

En general, la calidad del control prenatal que se brinda en Tijuana es satisfactoria y las mujeres que no reciben atención prenatal representan el grupo en mayor riesgo de tener hijos de menor peso al nacer. En 1992, $67601(1,7 \%)$ mujeres que fueron atendidas durante el parto en los Estados
Unidos no recibieron control prenatal, de un total de 3976509 mujeres atendidas (16). Cuando en una zona urbana como Tijuana $5,4 \%$ de las mujeres atendidas durante el parto carecen de control prenatal, la situación dista de ser óptima y revela una deficiencia en la atención maternoinfantil que exige un examen más profundo y una mayor promoción de la salud maternoinfantil entre las políticas de salud pública.

Durante la década pasada, la tasa de mortalidad infantil (de menores de un año) en México mostró un descenso significativo a expensas de la reducción de la mortalidad posneonatal (de niños de 1 a 12 meses de edad), mientras que la mortalidad neonatal (de menores de un mes) permaneció uniforme. Se observó un aumento de las malformaciones congénitas y trastornos perinatales, que en algunos estudios se han asociado con la calidad de la atención prenatal y obstétrica (17). Pese a la complejidad del problema, la mujer en alto riesgo de no recibir atención prenatal parece poseer ciertas características sociales y económicas definidas. De ahí que los programas de atención prenatal deban concentrarse en identificar a estas pacientes y en las que están en alto riesgo de sufrir problemas obstétricos. La falta de atención prenatal tiene implicaciones claras que llevan a la necesidad de colocar la atención prenatal de buena calidad al alcance de toda embarazada. Asimismo es necesario estudiar más a fondo los factores psicológicos y socioculturales que inciden en la falta de utilización del sistema de salud entre las mujeres embarazadas, aun cuando conocen su importancia, así como la posible influencia de la violencia doméstica en este fenómeno.

Agradecimiento. Se agradece a la Fundación Carnegie y a la Fundación Mexicana para la Salud el financiamiento proporcionado para este trabajo; a Raymundo Reyes su contribución al análisis estadístico; a Gilberto Robles Rivera y María Consuelo Segura su participación en la recolección de datos y a Helen Wallace sus ideas germinales en torno a este proyecto. 


\section{REFERENCIAS}

1. Nightingale EO, Soberón G, Peck MG, Mortimer AM. En ambos lados: investigación colaborativa binacional para mejorar la atención materno-infantil en la región de la frontera México-Estados Unidos. Border Health 1992; 3:4-6.

2. Zalar RW, McDuffie RS. Frequency of prenatal care visits and perinatal outcome [carta]. JAMA 1996;276:101.

3. Kotelchuck M, Delgado-Rodríguez M, GómezOlmedo M, Bueno-Cavanillas A, GálvezVargas R. Adequacy of prenatal care utilization. [Carta]. Epidemiology 1997;8:602-604.

4. Instituto Nacional de Estadística, Geografía e Informática. XI Censo General de Población y Vivienda, 1990. Tijuana: Consejo Estatal de Población de Baja California; 1992.

5. Luna RA, Rivera H. Mortalidad infantil en 1987 en la ciudad de Tijuana. Baja Medica 1989:1:21-24.

6. Institute of Medicine. The prevention of low birthweight: the report of the Committee to Study the Prevention of Low Birthweight. Washington, D.C.: Institute of Medicine; 1985.

7. Poland ML, Ager JW, Olson KL, Sokol RJ. Quality of prenatal care: selected social, be- havioral, and biomedical factors and birthweight. Obstet Gynecol 1990;75:607-611.

8. Korenbrot CC, Simpson L, Phibbs CS. Prenatal care needs assessment comparing service and outcomes in Fresno, California. Public Health Reports 1994;68-76.

9. Cobas JA, Balcazar H, Benin MB, Keith VM, Chong Y. Acculturation and low-birthweight infants among Latino women: a reanalysis of HHANES data with structural equation models. Am J Public Health 1996;86:394-396.

10. Byrd TL, Mullen PD, Selwyn BJ, Lorimor R. Initiation of prenatal care by low-income Hispanic women in Houston. Public Health Reports 1996;3:536-540.

11. Zambrana RE, Dunkel-Schetter C, Scrimshaw $S$. Factors which influence use of prenatal care in low income racial-ethnic women in Los Angeles County. J Community Health 191;16: 283-295.

12. Guendelman S, Jasis M. The health consequences of maquiladora work: women on the U.S.-Mexican border. Am J Public Health 1993;83:37-44.

13. Ditz PM, Gazmararian JA, Goodwin MM, Bruce FC, Johnson CH, Rochat W. Delayed entry to prenatal care: effect of physical violence. Obstet Gynecol 1997;90:221-224.

14. Alexander GR, Korenbrot CC. The role of pre natal care in preventing low birthweight. Future Child 1995;5:103-120.

15. Vega-Vega G. Evaluación de la atención maternoinfantil en cinco unidades de medicina familiar. Salud Publica Mex 1993;35: 283-287.

16. Elam-Evans LD, Adams MM, Gargiullo PM, Kiely JL, Marks JS. Trends in the percentage of women who received no prenatal care in the United States, 1980-1992: contributions of the demographic and risk effects. Obstet Gynecol 1996;87:575-580.

17. Vandale S, Rascón-Pacheco RA, Kageyama ML. Time-trends and causes of infant, neonatal and postneonatal mortality in Mexico, 1980-1990. Salud Public Mex 1997;39:48-51.

Manuscrito recibido el 3 de abril de 1998 y aceptado para publicación, tras revisión, el 27 de agosto de 1999.

ABSTRACT This study was intended to explore the conditions under which prenatal care is delivered in the border city of Tijuana, Baja California, Mexico, and to assess the possible associations between that care and neonatal results in terms of birthweight, health

\section{Prenatal care in the border city of Tijuana, Mexico} of the neonate, and prematurity. Seven hospitals serving persons from different socioeconomic strata were chosen, and between December 1993 and March 1994 interviews were conducted with 279 women who were in the first 24 to 48 hours of puerperium. During the interviews data were collected on socioeconomic level; the mothers' knowledge, attitudes, and practices concerning obstetric health; the mothers' perceptions of access to prenatal care; the quality of prenatal care visits (evaluated in terms of having blood and urine tested and weight and blood pressure measured); and the gynecological and obstetric and health history of the mother. A database was created using the SPSS statistics software package. Possible associations were explored, with prenatal care as the independent variable and various dependent variables, by means of contingency tables and a two-tailed Fisher's exact test.

None of the neonates was premature, ill, or had a birthweight of $\leq 2500 \mathrm{~g}$. For this reason it was decided to divide the variable corresponding to birthweight into two groupings, $\leq 3000 \mathrm{~g}$ and $>3000 \mathrm{~g}$. A significant $(P<0.00038)$ relationship was found between a lack of prenatal care and low birthweight. In addition, a lack of prenatal care was associated with: low family income; the mother's financial dependence on the father; the mother being in an unmarried relationship; little communication with the partner; having no medical insurance; an unwanted pregnancy; and giving delivery at the General Hospital. Out of the total sample of 279 women, 15 (5.4\%) had received no prenatal care. None of these 15 women reported they had encountered difficulties that prevented them from obtaining prenatal care, but only 7 of those women answered that question. The prenatal care was of good quality in 190 of the cases $(68 \%)$. Both the number and the timing of the prenatal visits were adequate in 142 of the cases (50\%). The mother's having worked during pregnancy or before had a positive predictive value in terms of utilization of prenatal care services. Additional studies are needed to evaluate the possible relationship between domestic violence and the lack of prenatal care or a delay in seeking such care. 